\title{
De l'exploitation familiale à la mobilisation collective
}

La place des conjointes dans un mouvement d'agriculteurs victimes des pesticides

From the Family Farm to a Collective Mobilisation. Wives in a Social Movement

of Farmers Suffering from Pesticide Poisoning

Jean-Noël Jouzel et Giovanni Prete

\section{(2) OpenEdition \\ Journals}

\section{Édition électronique}

URL : http://journals.openedition.org/travailemploi/7146

DOI : 10.4000/travailemploi.7146

ISSN : 1775-416X

\section{Éditeur}

DARES - Ministère du Travail

Édition imprimée

Date de publication : 1 juillet 2016

Pagination : 77-100

ISSN : 0224-4365

Référence électronique

Jean-Noël Jouzel et Giovanni Prete, « De l'exploitation familiale à la mobilisation collective », Travail et Emploi [En ligne], 147 । juillet-septembre 2016, mis en ligne le 11 juillet 2019, consulté le 05 septembre 2019. URL : http://journals.openedition.org/travailemploi/7146; DOI : 10.4000/travailemploi.7146 


\title{
De l'exploitation familiale à la mobilisation collective
}

\author{
La place des conjointes dans un mouvement \\ d'agriculteurs victimes des pesticides"
}

\author{
Jean-Noël Jouzel ${ }^{* *}$, Giovanni Prete ${ }^{* * *}$
}

\begin{abstract}
Dans cet article, nous étudions les liens entre les transformations à l'œuvre dans la sphère privée des familles d'agriculteurs et les modalités de la représentation publique de la profession agricole. Pour cela, nous nous interrogeons sur la place des femmes dans un mouvement d'agriculteurs qui s'estiment victimes des pesticides. Nous montrons qu'en tant que conjointes, elles constituent un appui décisif dans le parcours qui permet à leurs époux de se considérer et de se revendiquer comme des victimes des pesticides. Nous mettons également en évidence l'ambiguïté de leur positionnement dans l'action collective : reconnues comme d'indispensables relais de la mobilisation, elles restent essentiellement limitées à ce rôle d'intermédiaires, n'accédant ellesmêmes au statut de victimes qu'indirectement, par l'expérience du deuil. Si cette dernière légitime leur place au sein de l'association, elle induit également un éloignement vis-à-vis du monde agricole, générant d'inévitables tensions.
\end{abstract}

La sociologie rurale française connaît depuis une dizaine d'années un important renouvellement, dont un axe fort est l'étude des dynamiques de désenclavement social et politique qui caractérisent la profession agricole. Cette réflexion est particulièrement vive sur deux fronts de recherche. Le premier porte sur l'évolution des structures familiales paysannes, dont un ensemble de travaux quantitatifs et qualitatifs montre qu'elles tendent à s'aligner sur le reste de la société. Ces travaux mettent notamment en évidence la croissance de l'hétérogamie sociale des couples (RATTIN, 2002 ; GIRAUD, RÉMY, 2008), une augmentation du nombre de divorces (BESSIÈRE et al., 2008 ; BESSIÈRE, 2010), un allongement de la durée des études des enfants et une inscription de

\footnotetext{
* L'enquête sur laquelle repose cet article a bénéficié d'un financement par l'Office national de l'eau et des milieux aquatiques (Onema) dans le cadre de l'appel à projets de recherche en environnement-santé-travail lancé par l'Agence nationale de sécurité sanitaire de l'alimentation, de l'environnement et du travail (Anses) (APR Anses [EST-2012-135]). ** Centre de sociologie des organisations (CSO); jeannoel.jouzel@sciencespo.fr.

*** Institut de recherche interdisciplinaire sur les enjeux sociaux (Iris), université Paris 13 ; prete@ univ-paris13.fr.
} 
ces dernières dans des trajectoires de mobilité sociale (CHAMPAGNE, 2002 ; AlARCON, 2008 ; BESSIÈRE, 2010). Le second front de recherche s'intéresse à l'évolution des formes de représentation politique des agriculteurs. Plusieurs travaux donnent à voir la fragilisation du «mythe de l'unité paysanne », construit conjointement par les pouvoirs publics et par les syndicats d'exploitants durant les Trente Glorieuses (MARESCA, 1983 ; Muller, 1984), sous l'effet de représentations critiques vis-à-vis de l'orientation historique des politiques agricoles. La constitution d'une offre syndicale située sur la gauche de l'échiquier politique (BRUnEAU, 2013 ; HerviEu, PURSEIGLe, 2013) ainsi que l'institutionnalisation de pratiques alternatives aux choix techniques caractéristiques du productivisme, comme l'agriculture biologique (SAMAK, 2013 ; Hervieu, Purseigle, 2013), sont autant de facteurs de diversification des modes de représentation des agriculteurs.

Dans cet article, nous proposons d'analyser l'imbrication des dynamiques observées sur ces deux fronts de recherche, en nous intéressant aux effets des mutations des structures familiales sur les formes de représentation politique dans le milieu agricole. La sociologie des mouvements sociaux explore les liens entre relations familiales et engagement politique depuis plusieurs années, à travers notamment un intérêt de plus en plus marqué pour les problématiques de genre dans les mobilisations collectives (TAYLOR, 1999 ; EINWOHNER et al., 2000). Dans cette perspective, des travaux éclairent les déterminants genrés de l'engagement politique (DUNEZAT, 2006 ; Contamin, 2007), et notamment l'articulation entre trajectoires militantes et trajectoires familiales (FILliEule, 2009). Dans le champ de la sociologie rurale, l'étude du lien entre vie familiale et engagement politique est, cependant, longtemps restée marginale (LAGRAVE, 1983). Elle a surtout été abordée par des travaux portant sur le syndicalisme agricole, qui montrent que les luttes pour la représentation des agriculteurs ont pour objet la défense de certains modèles d'exploitation qui sont également des modèles d'organisation familiale (RÉMY, 1987) éventuellement adossés à des stéréotypes de genre (COMER, 2015). Ces travaux soulignent aussi parfois que les trajectoires syndicales des agriculteurs sont étroitement liées à leurs trajectoires familiales (Purseigle, 2004 ; Bruneau, 2007 ; Rieu, Fric, 2008).

Pour approfondir cette perspective de recherche, nous nous intéressons au rôle que tiennent les proches familiaux - et, principalement, les conjointes - dans la constitution de l'association Phyto-victimes. Cette dernière regroupe des travailleurs agricoles qui s'estiment victimes des pesticides après avoir contracté des pathologies chroniques graves repérées dans la littérature épidémiologique comme pouvant résulter de l'exposition professionnelle à ces produits (maladies de Parkinson, hémopathies malignes, etc. $)^{1}$. Malgré sa jeunesse ${ }^{2}$ et sa taille limitée ${ }^{3}$, Phyto-victimes présente

1. Pour une synthèse des connaissances disponibles sur les liens entre santé et expositions professionnelles aux pesticides, voir l'expertise collective de l'Institut national de la santé et de la recherche médicale (Inserm), Pesticides, effets sur la santé, publiée en 2013 ; en ligne : www.ipubli.inserm.fr/bitstream/handle/10608/4820/expcol_2013_pesticides. pdf?sequence $=1$, consulté le 8 mars 2016 .

2. Phyto-victimes a été créée en 2011.

3. L'association compte une dizaine de membres actifs et environ 200 adhérents. 
deux caractéristiques qui en font un terrain pertinent pour analyser la manière dont les évolutions de la structure familiale des agriculteurs travaillent les modalités de leur représentation politique. En premier lieu, cette association prétend s'imposer comme un représentant des intérêts des agriculteurs - et en particulier des exploitants - autonome et critique vis-à-vis des organisations professionnelles agricoles les plus établies, qu'elle met en cause pour avoir privilégié des orientations productivistes aux conséquences dommageables sur la santé humaine et l'environnement (JouzEL, PRETE, 2015). De plus, il s'agit d'une association de victimes, forme de mobilisation dont plusieurs travaux suggèrent qu'elle est particulièrement propice à l'observation des dynamiques d'interaction entre structure familiale et engagement politique, notamment quand elle investit l'enjeu des effets sanitaires des produits toxiques d'origine industrielle (Krauss, 1993 ; Brown, FERGUSON, 1995) (voir encadré 1 pour des précisions méthodologiques sur notre enquête).

Dans une première partie, nous mettons en évidence la contribution décisive des conjointes dans le parcours qui a permis à des agriculteurs malades de s'affirmer comme victimes des pesticides. Dans une deuxième partie, nous montrons que, si les conjointes sont reconnues en tant qu'appuis moraux et politiques importants par les victimes des pesticides, leur propre exposition aux produits toxiques n'est en revanche que partiellement reconnue comme enjeu problématique au sein de l'association. Plus généralement, nous soulignons comment la normalisation des structures familiales des exploitations agricoles favorise la mise en débat des effets du travail agricole sur la santé des agriculteurs, sans toutefois que la porosité entre leurs espaces professionnels et domestiques soit au cœur de ces débats. L'expérience du deuil associée à la perte d'un conjoint malade fait ici figure d'expérience-limite, qui permet aux femmes d'être à leur tour reconnues comme d'authentiques victimes des pesticides au sein de l'association, tout en induisant un éloignement vis-à-vis du monde agricole, générateur d'inévitables tensions qui fragilisent l'action collective.

\section{ENCADRÉ 1}

\section{Méthodologie de l'enquête}

Les données sur lesquelles repose cet article ont été produites dans le cadre d'une enquête qualitative dont l'objet principal a été l'émergence d'une mobilisation collective dénonçant les effets des pesticides sur la santé des travailleurs agricoles. Une vingtaine d'entretiens semi-directifs ont été menés avec des agriculteurs s'estimant malades du fait de leur exposition aux pesticides, une quinzaine d'entretiens ont été réalisés avec des tiers (médecins, avocats, journalistes, etc.) qui sont intervenus dans leur parcours, et une dizaine d'observations ont été conduites au sein de l'association Phyto-victimes (assemblées générales annuelles, conseil d'administration, manifestations publiques, etc.). L'enquête s'est particulièrement intéressée aux membres du conseil d'administration de Phyto-victimes, composé en grande partie des premiers travailleurs agricoles à avoir initié, en France, des procédures de reconnaissance de maladies professionnelles pour des pathologies imputables aux pesticides. 
Deux caractéristiques de l'enquête doivent être soulignées. D'une part, elle a principalement consisté à interroger des agriculteurs qui ont non seulement établi un lien entre leur pathologie et les pesticides, mais qui ont également considéré que ce lien pouvait être conçu comme un préjudice. Si nous avons aussi étudié des cas de travailleurs agricoles malades moins convaincus du lien entre leurs maux et les pesticides, ou du caractère préjudiciable de ce lien, nos données ne permettent pas de faire une comparaison systématique de leurs parcours avec ceux des membres de Phyto-victimes. D'autre part, cette enquête ne portait a priori pas principalement sur la place des conjointes des agriculteurs victimes dans les dynamiques de dénonciation collective des pesticides. Nos analyses s'en trouvent donc limitées, dans la mesure où des informations potentiellement intéressantes n'ont pas été systématiquement recueillies (par exemple, les récits de vie des conjointes d'agriculteurs victimes). Nous avons toutefois pu constituer un abondant matériau relatif à la place des conjointes dans l'action collective grâce à l'observation de cette dernière, mais également grâce aux entretiens : initialement prévus avec le seul agriculteur victime, ils se sont presque toujours transformés en entretiens de couple, la conjointe - et, parfois, les enfants - se trouvant conviés à y participer à un moment ou à un autre.

\section{Le rôle déterminant des conjointes pour les agriculteurs victimes des pesticides}

Dans les décennies qui ont précédé et suivi la Seconde Guerre mondiale, l'agriculture française s'est de plus en plus orientée vers un modèle productiviste qui, caractérisé par une recherche permanente d'augmentation des rendements à l'hectare et par l'agrandissement des exploitations, s'est accompagné d'une diminution de leur nombre (DESRIERS, 2007). Les intrants chimiques (engrais et pesticides) ont constitué l'un des pivots technologiques permettant cette mutation sociotechnique. Les quantités de produits phytosanitaires de synthèse épandus dans les exploitations françaises ont ainsi spectaculairement augmenté des années 1960 aux années 1980. Les effets de ces produits, par définition toxiques, sur la santé des agriculteurs exposés sont longtemps restés invisibles, notamment en raison d'importants temps de latence séparant l'exposition de la survenue de maladies chroniques susceptibles d'en résulter. Depuis une vingtaine d'années, des données épidémiologiques issues de la littérature internationale et française convergent pour attribuer à l'exposition des travailleurs agricoles aux pesticides la surincidence dans cette population de certaines pathologies chroniques telles que des maladies neurodégénératives (principalement la maladie de Parkinson) et certains cancers (en particulier du système sanguin). En mars 2011, une poignée d'agriculteurs ayant entamé, et parfois conclu avec succès, des démarches de reconnaissance de maladies professionnelles pour des pathologies de ce type ont fondé l'association Phyto-victimes. L'étude de la genèse de cette association montre le rôle décisif qu’y ont joué les épouses de ces agriculteurs malades. 


\section{Une affaire d'hommes... très entourés}

L'association Phyto-victimes revendique aujourd'hui environ 200 adhérents. Son fonctionnement repose cependant essentiellement, depuis sa création, sur l'implication des membres de son conseil d'administration, dont le tableau suivant présente une liste anonymisée (de sa composition initiale), accompagnée de quelques précisions sur leur activité professionnelle et sur leur situation familiale.

\section{TABLEAU - Présentation synthétique des profils des membres du conseil d'administration de Phyto-victimes à la création de l'association}

\begin{tabular}{|c|c|c|c|c|}
\hline Prénom & Préjudice subi & Activité & Situation familiale & $\begin{array}{l}\text { Profession du/de la } \\
\text { conjoint.e (ou de l'ex- } \\
\text { conjoint.e en cas de } \\
\text { divorce ou de décès) }\end{array}$ \\
\hline (1) Jean-Yves & Cancer & Salarié de coopérative & Divorcé avec enfants & Enseignante \\
\hline (2) Bertrand & Cancer & $\begin{array}{l}\text { Exploitant, polyculture, } \\
\text { élevage (allaitantes) }\end{array}$ & Marié avec enfants & Secrétaire médicale \\
\hline (3) Magalie & $\begin{array}{l}\text { Veuve d'un exploitant } \\
\text { décédé d'un cancer }\end{array}$ & $\begin{array}{l}\text { Ex-comptable, devenue } \\
\text { exploitante, polyculture, } \\
\text { élevage (allaitantes) }\end{array}$ & Veuve avec enfants & $\begin{array}{l}\text { Exploitant, polyculture- } \\
\text { élevage (allaitantes) }\end{array}$ \\
\hline (4) Roger & Cancer & $\begin{array}{l}\text { Exploitant, polyculture, } \\
\text { maraîchage }\end{array}$ & Marié avec enfants & Exploitante agricole \\
\hline (5) François & $\begin{array}{l}\text { Père d'un exploitant } \\
\text { décédé d'un cancer }\end{array}$ & $\begin{array}{l}\text { Technicien agricole et } \\
\text { viticulteur retraité }\end{array}$ & Marié avec enfants & Exploitante agricole \\
\hline (6) Jacques & $\begin{array}{l}\text { Problèmes } \\
\text { neurologiques }\end{array}$ & $\begin{array}{l}\text { Exploitant, } \\
\text { céréaliculture }\end{array}$ & Marié avec enfants & $\begin{array}{l}\text { Directrice d'une maison } \\
\text { de retraite }\end{array}$ \\
\hline (7) René & $\begin{array}{l}\text { Problèmes } \\
\text { neurologiques }\end{array}$ & $\begin{array}{l}\text { Exploitant, } \\
\text { céréaliculture bio }\end{array}$ & Marié avec enfants & Exploitante agricole \\
\hline (8) Christian & Cancer & $\begin{array}{l}\text { Exploitant, } \\
\text { céréaliculture, élevage } \\
\text { (laitières) }\end{array}$ & Marié avec enfants & $\begin{array}{l}\text { Chômage, ex-secrétaire } \\
\text { de direction d'une PME" }\end{array}$ \\
\hline (9) Yvette & $\begin{array}{l}\text { Veuve d'un exploitant } \\
\text { décédé d'un cancer }\end{array}$ & Secrétaire de mairie & Veuve avec enfants & $\begin{array}{l}\text { Exploitant, polyculture- } \\
\text { élevage (allaitantes) }\end{array}$ \\
\hline (10) Hugues & Parkinson & $\begin{array}{l}\text { Exploitant, viticulture, } \\
\text { polyculture, élevage } \\
\text { (allaitantes) }\end{array}$ & Marié avec enfants & $\begin{array}{l}\text { Secrétaire de centre } \\
\text { financier }\end{array}$ \\
\hline (11) Patrick & Parkinson & $\begin{array}{l}\text { Retraité, ancien } \\
\text { exploitant puis salarié } \\
\text { agricole }\end{array}$ & Marié avec enfants & Institutrice \\
\hline
\end{tabular}

* Petite et moyenne entreprise.

Note : l'ensemble des prénoms utilisés pour désigner les personnes interrogées au cours de notre enquête sont fictifs.

Deux constats s'imposent à la lecture de ce tableau. En premier lieu, bien qu'ouverte potentiellement à tous les travailleurs agricoles, l'association est animée principalement par des exploitants, statutairement indépendants. Tous ces chefs 
d'exploitation ont pratiqué une agriculture conventionnelle et intensive à la tête de fermes de taille relativement importante et continuent leur activité, pour certains en dépit de handicaps lourds dus aux pathologies dont ils ont souffert. Seuls trois salariés agricoles participent au conseil d'administration, et tous ont des liens familiaux avec des exploitants ou l'ont eux-mêmes été. Le premier, François, technicien agricole à la retraite, est le conjoint d'une exploitante viticole près de Cognac; leur fils, qui a repris l'exploitation familiale, est mort d'un cancer. Le deuxième, Patrick, ancien chef de culture en région Centre-Val de Loire, souffrant de la maladie de Parkinson, était auparavant lui-même exploitant céréalier. Le troisième représentant salarié, Jean-Yves, est un ancien technicien de coopérative, atteint d'un cancer, fils et petit-fils d'exploitants viticoles et agricoles du sud-est de la France. En somme, et même si elle entend représenter l'ensemble des agriculteurs victimes des pesticides quel que soit leur statut, Phyto-victimes est d'abord une association d'exploitants.

Deuxièmement, à première vue, l'association Phyto-victimes semble être une affaire d'hommes. Neuf de ses onze membres fondateurs sont de sexe masculin. Quant aux deux femmes, elles ne sont pas présentes en tant qu' agricultrices victimes des pesticides, mais en tant que veuves d'agriculteurs dont le décès est imputé à l'exposition à ces produits. L'observation de la vie de l'association permet néanmoins de nuancer ce constat d'une omniprésence masculine. Outre le fait que l'association a recruté deux femmes chargées de mission, presque tous les hommes membres du conseil d'administration sont régulièrement entourés par leur conjointe et, plus largement, par leur famille. La plupart sont mariés de longue date et ont des enfants. Il n'est pas rare que leur conjointe, leurs enfants ou leurs parents les accompagnent dans les différents événements qui rythment la vie de l'association. Si, dans certains cas, la présence des proches se limite à l'apparition ponctuelle d'une épouse ou d'un enfant lors d'une assemblée générale annuelle, de manifestations publiques ou d'une prise de parole médiatique, dans d'autres, leur engagement va nettement plus loin. C'est notamment le cas pour plusieurs conjointes. Trois des épouses des membres du conseil d'administration de Phyto-victimes assistent ainsi quasi systématiquement à ses réunions et y prennent parfois la parole sans en être pour autant officiellement membres. L'association Phyto-victimes est donc à la fois une affaire d'hommes, de familles et de couples. Cette situation n'est pas le fruit du hasard, elle est le produit de l'engagement des proches familiaux, et particulièrement des conjointes, dans le parcours victimaire des agriculteurs les plus actifs au sein de Phyto-victimes.

\section{Les conjointes : un appui décisif dans les parcours des agriculteurs victimes des pesticides}

La notion de parcours victimaire renvoie au caractère progressif de l'engagement des travailleurs agricoles dans la cause politique des victimes des pesticides. Se reconnaître et être reconnu victime des pesticides implique en effet le franchissement d'étapes communes à la plupart des trajectoires de victimes de maladies professionnelles pour 
lesquelles des produits toxiques sont mis en cause : prendre conscience du lien potentiel entre la maladie et l'exposition au toxique et du préjudice entraîné par la pathologie, chercher à obtenir une compensation matérielle ou symbolique du tort subi, militer dans une association dénonçant publiquement les dangers et les responsabilités en jeu ${ }^{4}$. Dans le cas des agriculteurs ayant fondé Phyto-victimes, les conjointes jouent un rôle décisif dans toutes les étapes du processus.

La plupart des agriculteurs interrogés ont pris conscience d'un lien possible entre la dégradation de leur état de santé et l'exposition aux pesticides lors d'échanges avec le corps médical (médecine spécialisée et/ou médecine du travail), au cours d'un processus itératif qui a peu à peu fait émerger l'étiologie de leurs pathologies. Outre que les médecins n'explorent pas systématiquement les liens entre travail et santé, en particulier en ce qui concerne les expositions aux toxiques (THÉBAUD-Mony, 2006), les connaissances quant aux dommages éventuels causés par les pesticides sur la santé étaient, au moment où les personnes que nous avons rencontrées sont tombées malades, faiblement diffusées, y compris dans les cercles les plus spécialisés du corps médical (hématologie, neurologie $)^{5}$. Dans ce contexte, les conjointes ont parfois joué un rôle important pour mettre les agriculteurs et leurs médecins sur la piste des pesticides. Plusieurs d'entre elles ont été très présentes dans le parcours médical de leurs maris, assistant aux visites et y prenant éventuellement la parole pour évoquer cette hypothèse parmi d'autres.

Une fois la connexion causale entre pathologie et exposition aux pesticides établie, l'entrée des exploitants dans un processus d'indemnisation via une demande de reconnaissance de maladie professionnelle a été largement encouragée, voire initiée, par leur épouse. Dans la plupart des couples enquêtés, ce sont elles qui ont pris en charge le montage et le suivi du dossier de reconnaissance (envois et lectures de courriers, relances adressées à l'administration, recherche d'informations juridiques, etc.). D'ailleurs, lors de la majorité des entretiens, l'épouse, absente au début de l'interaction, a été conviée par son mari à y participer dès lors que nous demandions à ce dernier de dater son parcours victimaire ou d'en produire des archives. Pour convaincre leur époux d'engager une demande de reconnaissance de maladie professionnelle, ces femmes ont souvent eu à lever des réticences initiales de plusieurs ordres. Tout d'abord, chefs d'exploitation indépendants ${ }^{6}$ ayant longtemps assuré les opérations de traitement aux pesticides sans toujours se protéger, les agriculteurs concernés se sont parfois perçus comme principaux responsables de leurs malheurs. Une telle perception a été encouragée par les messages de prévention qu'ont reçus les agriculteurs. Mettant en avant le caractère « contrôlé » (DÉCOSSE, 2013) des risques découlant de l'utilisation

\footnotetext{
4. On reconnaît ici les catégories employées par William L. F. FeLSTINER et ses coauteurs (1980-1981) pour décrire l'évolution des litiges, à partir, notamment, du cas des travailleurs victimes de l'amiante. Pour une lecture plus serrée de cette référence, voir JouZEL, PRETE, 2013.

5. La publication en 2013 du rapport de l'Inserm, mentionné plus haut, sur les liens entre pesticides et santé a rendu plus facilement disponibles les données épidémiologiques et toxicologiques produites depuis les années 1980.

6. Sur les limites de l' « indépendance » des exploitants et de l'autonomie de leurs choix techniques et économiques, voir LORVELLEC, 1998.
} 
des pesticides, ces messages enjoignent aux exploitants d'adopter, individuellement, de $«$ bonnes pratiques » agricoles $^{7}$ :

«-Votre mari était très moteur ou... [pour faire une démarche de reconnaissance en maladie professionnelle]?

Magalie : - Non, c'est moi qui étais moteur, moi et [une amie].

- Il en disait quoi, lui ?

- Il était très malade, et puis ça le mettait dans une position un peu compliquée, par rapport au fait qu'il ne se soit pas protégé. Pour lui, il était responsable de sa propre maladie. Dans sa tête c'était assez compliqué, c'était... En même temps, la reconnaissance en maladie professionnelle a permis d'embaucher un salarié à temps complet (avant il était à mi-temps), et de pouvoir le remplacer complètement sur l'exploitation ; ça pour lui c'était important. Mais en même temps, cette reconnaissance, c'était vraiment : "Je me suis empoisonné tout seul." Lui ne s'est pas positionné en disant : “C'est la responsabilité des firmes" ; il s'est dit : "Merde, j'ai déconné, je ne me suis pas protégé, c'est ma faute." »

(Entretien avec Magalie ${ }^{8}$, membre du conseil d'administration de Phyto-victimes, veuve d'un exploitant décédé, juillet 2012)

Ensuite, les agriculteurs ont pu être récalcitrants à l'idée de s'engager dans des démarches complexes ${ }^{9}$, qu'ils connaissaient mal $^{10}$, et aux résultats incertains alors même qu'ils étaient malades et que leur premier souci était de s'assurer que le travail nécessaire à la survie de l'exploitation était réalisé. Enfin, il est possible qu'ils aient craint les conséquences négatives d'un tel processus sur d'autres aspects de leur vie professionnelle. Par exemple, un agriculteur nous a expliqué redouter que l'éventuelle qualification de son affection en maladie professionnelle entraîne le retrait de son permis de conduire. Un autre nous a confié appréhender que des informations médicales personnelles le concernant soient divulguées, l'empêchant à l'avenir de décrocher un prêt bancaire. Ces craintes, même quand elles sont peu fondées, découragent les agriculteurs d'entreprendre une demande d'indemnisation. Or leurs épouses ont parfois

\footnotetext{
7. Sans entrer dans le détail de ce point traité plus avant ailleurs (JouZEL, DediEU, 2013), rappelons simplement que la mise sur le marché des produits phytopharmaceutiques s'adosse à la promotion de prescriptions relatives aux « bonnes pratiques agricoles » et au port d'équipements de protection individuels (EPI : gants, combinaison, masque). Moyennant le respect de ces précautions, l'agriculteur est censé n'être exposé qu'à une dose ne mettant pas en péril sa santé. Ces préconisations ont pour conséquence de rendre les exploitants responsables de leur propre protection alors même que les informations sur la dangerosité des produits inscrites sur les étiquettes ont longtemps été lacunaires. De plus, des doutes ont été émis à la fois sur l'efficacité des EPI et le fait qu'ils soient adaptés aux réalités du travail agricole (GARRIGOU et al., 2008).

8. Nous rappelons que l'ensemble des prénoms utilisés pour désigner les personnes interrogées au cours de notre enquête sont fictifs.

9. Jusqu'en 2012, il n'existait que très peu de tableaux de maladies professionnelles permettant une reconnaissance « automatique » du lien entre l'exposition d'un travailleur agricole aux pesticides et une pathologie susceptible d'en découler. Les victimes ou leurs ayants droit devaient donc systématiquement passer par une procédure longue et complexe (comités régionaux de reconnaissance des maladies professionnelles, tribunaux des affaires de Sécurité sociale) (JouZEL, PreTe, 2014a).

10. Ce n'est que depuis 2002 qu'a été mise en place une assurance obligatoire pour les accidents du travail et les maladies professionnelles pour les exploitants agricoles (JOUZEL, PrETE, 2014b).
} 
réussi à vaincre leurs réserves, notamment en faisant valoir l'intérêt de se lancer dans un processus de reconnaissance en maladie professionnelle pouvant se conclure par l'obtention d'une rente qui améliorerait la situation matérielle de l'exploitation et de la cellule familiale. Comme nous l'avons observé plus particulièrement dans certains cas, les épouses jouent un rôle moteur dans les démarches d'indemnisation (encadré 2).

Enfin, certaines épouses ont incité leur mari à tenter d'identifier et de dénoncer les tiers responsables de leurs maux. Aujourd'hui, les membres actifs de Phyto-victimes articulent étroitement démarche de reconnaissance de maladies professionnelles et

\section{ENCADRÉ 2}

\section{La famille, un soutien face à la maladie et aux difficultés du parcours de reconnaissance}

Bertrand est agriculteur dans le Jura. En 1987, son bac en poche et après deux ans comme « aide familial », il s'installe sur la petite exploitation en polyculture élevage de ses parents qui prennent leur retraite. Après leur décès, il modernise fortement l'exploitation : agrandissement, mécanisation, remplacement d'un élevage laitier par celui de vaches allaitantes... et recours accru aux produits chimiques (son père n'utilisait des désherbants qu'en petite quantité). Il acquiert donc l'équipement adéquat, ce qui lui permet notamment de faire des épandages en prestation de service auprès de voisins en se croyant protégé. En 2008, souffrant d'un mal de dos lancinant, il consulte plusieurs médecins qui diagnostiquent un cancer et une tumeur sur la colonne vertébrale. Opéré en urgence, il met plusieurs mois à guérir de son cancer mais reste hémiplégique. Pour faire face à la maladie, il bénéficie du soutien de proches. Des amis et des membres de sa famille (l'une de ses sœurs est mariée avec un agriculteur) l'aident à s'occuper de son exploitation pendant toute la période durant laquelle il effectue de nombreux allers et retours à l'hôpital, où il suit une chimiothérapie puis un programme de rééducation. Sa femme, Martine, qu'il a rencontrée la dernière année de ses études, joue également un rôle clé. Bien avant que sa maladie ne se déclare, elle s'inquiète de le voir faire de l'épandage de produits chimiques aussi fréquemment, sur son exploitation comme sur celles des voisins. Une fois le cancer diagnostiqué, elle le soutient moralement, s'occupe de leurs deux jeunes garçons et l'accompagne à ses multiples rendez-vous médicaux. Elle l'aide aussi à faire reconnaitre l'origine professionnelle de sa maladie. Titulaire d'un BTS ${ }^{1}$ de secrétaire médicale, ayant travaillé dans une clinique radiologique et dans un Centre hospitalier, elle participe au montage de son dossier et le pousse à mener jusqu' au bout, malgré des découragements réguliers, les démarches ${ }^{2}$ qu'ils décident d'engager après qu'un médecin évoque un lien possible entre les pesticides utilisés par Bertrand et sa maladie. En 2011, l'origine professionnelle de sa maladie est reconnue. Son épouse l'accompagne à toutes les réunions de Phyto-victimes.

1. Brevet de technicien supérieur.

2. Passage devant le comité régional de reconnaissance des maladies professionnelles (CRRMP), prise de contact avec un avocat, passage devant le tribunal des affaires de sécurité sociale (Tass). 
dénonciation des entreprises productrices de pesticides, qu'ils accusent notamment de dissimuler les dangers des produits qu'elles mettent sur le marché ${ }^{11}$. Notre enquête montre que l'indignation à l'encontre des fabricants de pesticides n'est jamais présente au début du parcours des victimes. Toutes étaient alors très éloignées de l'écologie politique, et peu défiantes vis-à-vis de l'industrie chimique en général, et de l'industrie phytopharmaceutique en particulier. Leur colère est peu à peu montée, notamment au fil de rencontres avec des acteurs critiques des effets sanitaires des polluants industriels : militants environnementalistes, scientifiques, journalistes, avocats, etc. Sur ce point, que nous avons analysé en détail dans une autre publication (JouZEL, PreTE, 2014a), soulignons simplement ici que les épouses des exploitants ont souvent été à l'initiative de ces rencontres, permettant ainsi à leur mari de pleinement s'informer sur les dangers des pesticides.

\section{Le poids de l'évolution des structures familiales dans l'engagement militant}

Le rôle central des conjointes dans les parcours de victimes des pesticides mérite d'être mis en perspective avec l'évolution des structures familiales agricoles sur au moins deux aspects.

Le premier renvoie à l'hétérogamie croissante des familles d'exploitants. Jadis érigé en norme sociale, dans le cadre de la promotion politique et économique d'un « modèle productiviste dans un cadre familial » (BESSIÈRE, 2011), le modèle du couple d'exploitants est en perte de vitesse rapide (Dufour, GiRAud, 2012). S'impose, notamment parmi les jeunes générations d'agriculteurs, un modèle de couple composé d'un conjoint exploitant, généralement un homme, et d'un conjoint, généralement une femme (RATTIN, 2002), appartenant à la catégorie des employés ou des professions intermédiaires (DUBUISSON, GIRAUD, 2010). En outre, ces conjointes d'exploitants qui travaillent à l'extérieur de l'exploitation sont de plus en plus fréquemment issues de milieux non agricoles (GIRAUD, RÉMY, 2008). Le conseil d'administration de l'association Phyto-victimes rassemble des familles aux profils particulièrement typiques au regard de ces évolutions : il est majoritairement composé d'exploitants en couple avec des conjointes travaillant ou ayant travaillé dans le secteur tertiaire, dont la plupart n'ont pas grandi sur une exploitation agricole. Dans le tableau présenté plus haut, seul le couple formé par Roger et son épouse (ligne 4) correspond au modèle productiviste dans un cadre familial (celui formé par René et sa femme, décrit ligne 7, s'en rapproche, mais exploite une ferme en agriculture biologique). Ce positionnement social en dehors de l'agriculture a ouvert à ces femmes l'accès à des ressources précieuses pour faire avancer leur conjoint dans leurs parcours victimaires, depuis la prise de conscience du lien entre leur maladie et les pesticides jusqu'à l'entrée dans un mouvement collectif de dénonciation de ces produits. Nos observations contrastent avec les résultats des travaux nord-américains sur les mouvements d'environmental justice (KRAUSS,

11. Sur l'apparition des logiques de dénonciation dans les trajectoires de victimes, voir BARBOT, FILLION, 2007. 
1993 ; Brown, Ferguson, 1995), qui soulignent que l'implication importante des femmes dans les mobilisations collectives dénonçant les effets sanitaires des produits industriels toxiques constitue souvent un prolongement de leur position de mères de famille dans l'espace domestique, puisqu'elles y sont notamment en charge de la surveillance de l'état de santé des membres de la famille en général et des enfants en particulier ${ }^{12}$. Dans le cas de l'association Phyto-victimes, c'est surtout la position occupée par l'épouse à l'extérieur du foyer, parfois même loin de l'exploitation, qui semble déterminante dans son propre engagement en vue de faire reconnaître le statut de victime de son conjoint (encadré 3).

Le deuxième aspect de l'évolution des structures familiales agricoles renvoie aux mutations des relations intergénérationnelles, qui s'expliquent notamment par les

\section{ENCADRÉ 3}

\section{Hors de l'exploitation, une épouse sur la piste des pesticides}

Après des études agricoles courtes, Christian intègre le groupement agricole d'exploitation en commun (Gaec) familial en 1978, en Lorraine. Il rejoint son père et deux de ses frères sur une grosse exploitation de plus de 350 hectares, principalement dédiée à la céréaliculture ; il y est chargé de faire quasiment tous les traitements. En 1983, il épouse Laurence, avec qui il a deux enfants, un fils et une fille, dont aucun n'envisage aujourd' hui de reprendre l'exploitation. Laurence occupe différents postes de secrétariat et fait des remplacements occasionnels en tant que conductrice dans l'entreprise d'autocars de ses parents. Pour faciliter ces remplacements, le couple emménage en ville, à 50 kilomètres de l'exploitation. La distance géographique avec l'activité agricole semble avoir contribué à rendre plus évidents aux yeux de Laurence les risques induits par les produits phytosanitaires. Tous les soirs, elle constate que son mari rentre à leur appartement en ville en «puant les pesticides ». En 2002, le diagnostic tombe : Christian est atteint d'un syndrome myéloprolifératif. Laurence, suspectant les pesticides qu'il a épandus d'en être la cause, interroge les médecins et son entourage sur cette hypothèse, et encourage fortement son mari à déposer un dossier de reconnaissance en maladie professionnelle. Cette démarche devient son combat. Elle engage les services d'une avocate qu'elle côtoie dans le cadre de son travail, constitue le dossier administratif et prend contact avec des acteurs déjà engagés dans la dénonciation des dangers des pesticides, comme le président de la principale association environnementale militant pour l'interdiction massive de ces produits. Si ces prises de contact s'inscrivent d'abord dans la perspective d'une collecte d'informations destinées à consolider le dossier de reconnaissance de son conjoint et à faire face aux difficultés matérielles liées à la maladie (par exemple la perte de capacité d'endettement), elles prennent progressivement une tournure plus politique et posent les jalons de l'implication à venir du couple dans la création de Phyto-victimes en 2011.

12. On trouvera une critique de la tendance de certains de ces travaux à surévaluer le lien entre engagement politique, investissement dans le foyer et maternité, et, de ce fait, à paradoxalement essentialiser les ressorts de l'engagement des femmes dans PERKINS, 2012. 
choix d'orientations scolaires des enfants d'exploitants. Les travaux sur cette question soulignent qu'une grande partie des enfants d'agriculteurs ne se destinant pas aux métiers de l'agriculture a un parcours scolaire normalisé (CHAMPAGNE, 2002 ; ALARCON, 2008 ; GiRAUd, RÉMY, 2015). Encore une fois, les familles des membres du conseil d'administration de Phyto-victimes sont assez représentatives de ces évolutions. Leurs enfants ne souhaitent majoritairement pas travailler dans l'agriculture. Or le fait que son exploitation ait toutes les chances de ne pas être reprise par l'un de ses enfants peut précisément inciter un agriculteur à s'engager dans un parcours victimaire. Il craint alors nettement moins les conséquences de représailles symboliques ou matérielles dont il pourrait faire les frais s'il venait à s'interroger publiquement - lors d'interviews dans la presse, d'apparitions dans des films ou de participations à des actions médiatisées de l'association - sur le caractère indispensable des pesticides dans l'activité agricole. Au sein de l'association, des anecdotes circulent qui alimentent les inquiétudes à ce sujet. Un membre du conseil d'administration attribue par exemple des actes malveillants répétés sur les clôtures de son exploitation à la médiatisation de ses démarches médicoadministratives. Qu'elles soient ou non justifiées, ces inquiétudes peuvent avoir un impact sur les parcours d'agriculteurs malades. L'anecdote suivante, relatée dans un entretien, montre par exemple comment ce type de craintes peut bloquer des démarches de reconnaissance. L'interviewé évoque une famille qui a préféré y renoncer plutôt que de courir le risque de se voir accusée par l'environnement professionnel local (organisations agricoles et voisins) de mauvaise publicité faite au « monde agricole » :

« J'ai rencontré à Limoges deux familles. Puis une en particulier, le mari est décédé, quoi. [À] la femme et la fille, j' ai dit : "Écoutez, on doit pouvoir vous aider." On sent que c'est extrêmement compliqué. Les médecins leur ont dit qu'il fallait pas chercher. Une leucémie foudroyante comme ça, fallait pas chercher [sous-entendu : le lien avec les pesticides est évident]. Et [un responsable d'une association limousine luttant contre les épandages de pesticides] leur dit : "Faut y aller", enfin... "faut aller vers l'association, et puis il faut entamer une procédure parce que vous avez des droits" ; et puis elles ont dit clairement : "Notre fils il a repris l'exploitation, tout ça, et on veut pas qu'il ait d'ennuis." »

(Entretien avec Jacques, exploitant agricole membre du conseil d'administration de Phyto-victimes, mars 2013)

\section{Si proches, si loin : centralité et périphérie des femmes dans la cause des Phyto-victimes}

Les conjointes des exploitants membres de Phyto-victimes ont joué un rôle déterminant dans le parcours qui a conduit ceux-ci à s'indigner de leur intoxication par les pesticides et à s'engager dans la construction d'une cause politique. Pour cette raison, elles participent de manière décisive au fonctionnement de l'association depuis sa création. La place centrale des femmes dans l'action collective ne doit cependant pas 
masquer le caractère partiel de la reconnaissance qui leur y est accordée : si elles sont largement mises en avant dans les prises de position publiques de Phyto-victimes, c'est principalement en tant qu'épouses accompagnantes et non en tant que victimes potentielles des pesticides. Il en résulte des tensions quand ces femmes revendiquent au sein de l'association un autre rôle que celui d'indispensables relais du parcours victimaire, notamment lorsqu'elles traversent l'épreuve du deuil après la perte de leur conjoint malade.

\section{L'expérience féminine de la maladie : entre naturalisation et mise en scène}

Comme nous l'avons signalé plus haut, plusieurs épouses d'agriculteurs malades prolongent sur le terrain militant leur engagement dans le parcours de reconnaissance du statut de victime de leur conjoint. Si leur rôle au sein de l'association Phyto-victimes n'est généralement pas officialisé par une élection au conseil d'administration, elles n'en participent pas moins aux activités de ce dernier et à l'orientation de l'action collective. Pour les autres membres de Phyto-victimes, cette participation a longtemps ${ }^{13}$ relevé d'une forme d'évidence non questionnée, légitimée par une vision genrée des attitudes féminines et masculines vis-à-vis des maladies imputables aux pesticides. Dans cette perspective, il va de soi que ce sont essentiellement des hommes qui souffrent de ces maladies, mais que les femmes sont davantage capables de s'en indigner et d'en faire un objet de mobilisation personnelle et collective. Aux yeux des membres masculins du conseil d'administration, les femmes sont d'autant plus nécessaires à la cause qu'elles n'ont « forcément, pas la même sensibilité qu' [eux ] $]^{14}$ » vis-à-vis de leurs propres souffrances.

La centralité des femmes dans l'action revendicatrice de Phyto-victimes est nettement visible dans plusieurs manifestations organisées par l'association. Dès la création de cette dernière, les conjointes sont en effet délibérément mises en avant. C'est dans cet esprit qu'est par exemple envisagée, en février 2012, l'une des premières actions d'envergure de Phyto-victimes, au Salon de l'agriculture à Paris. Les membres du conseil d'administration de l'association décident d'occuper le stand de l'Union des industries de la protection des plantes (UIPP), qui regroupe les principaux fabricants de pesticides, en demandant à « treize veuves d'agriculteurs de porter chacune un t-shirt avec une des lettres de Phyto-victimes », non seulement parce qu'elles "portent la souffrance » et que ce sont souvent elles qui « ont entamé les démarches », mais aussi parce qu'elles « apportent un témoignage » et qu'elles ont un « discours fort ${ }^{15} »$. Si ce projet est abandonné devant la difficulté à trouver un nombre suffisant de femmes

\footnotetext{
13. Au cours de l'année 2016, la participation des épouses au conseil d'administration de Phyto-victimes a cependant fait l'objet de questionnements émergents au sein de l'association. Trop récents pour être analysés dans le cadre de cet article, ces questionnements appellent un travail empirique plus approfondi.

14. Intervention de Christian, exploitant atteint d'un syndrome myéloprolifératif, lors du conseil d'administration de Phyto-victimes du 15 mars 2016.

15. Extraits de notes prises lors d'une réunion préparatoire de cet événement en décembre 2011. Sur la mise en scène des émotions dans les mouvements sociaux, on pourra se reporter à plusieurs contributions dans TRAÏNI (dir.), 2009.
} 
d'exploitants décédés acceptant de témoigner, la veuve d'un agriculteur est néanmoins chargée de lire un discours au nom des «femmes d'agriculteurs » :

« Nous, femmes d'agriculteurs, prenons la parole aujourd'hui devant l'UIPP. Nos maris sont morts des suites de leurs maladies, d'autres sont gravement malades. Dans tous les cas le lien entre leur pathologie et l'utilisation de produits phytosanitaires a été reconnu. Souvent après de longues démarches et des procédures devant les tribunaux. À travers l'association Phyto-victimes, nous sommes là pour dire à tous nos collègues agriculteurs et à toutes les professions qui utilisent ou ont utilisé des pesticides qu'il y a un danger pour leur santé. »

(Retranscription du discours tenu à l'occasion du Salon de l'agriculture, février 2012)

Par la suite, l'aide que les conjointes apportent à leur mari continue à être publiquement soulignée par les membres actifs de l'association. En juin 2015, la soirée qui suit l'assemblée générale est dédiée aux «femmes qui soutiennent Phyto-victimes » pour leur « rendre hommage » et saluer leur investissement dans les démarches médico-administratives ou dans les luttes politiques. Si cette insistance sur l'aide et la sensibilité des femmes traduit les conceptions genrées du rôle des femmes, dominantes au sein de l'association, elle est également le fruit d'un investissement stratégique, les hommes comme les femmes de l'association percevant nettement que ces dernières sont des porte-voix efficaces de la cause ${ }^{16}$.

\section{Les victimes au masculin?}

Pour autant, la place qu'occupent les femmes dans le fonctionnement de l'association comme dans ses manifestations publiques est ambiguë. Quel que soit leur sexe, les membres de Phyto-victimes les voient principalement comme des épouses accompagnantes, et ne se posent que très peu la question de leur éventuel statut de victimes des pesticides. Si plusieurs enquêtes démontrent que le partage des tâches sur une exploitation agricole obéit à une logique genrée (BARTHEZ, 1982 ; RIEU, 2004 ; GIRAUD, RÉMY, 2013) et suggèrent que les traitements phytosanitaires sont, en tant qu'opérations techniques jugées stratégiques pour l'exploitation, principalement réalisés par des hommes (typiquement le chef d'exploitation), rien ne prouve cependant que l'exposition aux pesticides des femmes d'agriculteurs, qu'elles travaillent ou non sur l'exploitation, soit négligeable. De fait, dans l'univers professionnel agricole, les contacts avec les pesticides ne se limitent pas aux expositions « directes » induites par les tâches afférentes aux traitements des cultures (préparation de la bouillie, épandage, nettoyage du matériel de traitement). De nombreux travaux agricoles occasionnent des expositions indirectes aux pesticides, et ces travaux sont souvent effectués par des femmes : tâches de taille ou de récolte imposant de « ré-entrer » sur une parcelle traitée

16. Ce double registre, naturalisé et stratégique, de justification de l'engagement différencié des participants à l'action collective dans l'espace public en fonction de leur sexe a déjà été observé dans d'autres travaux. Voir en particulier EINWOHNER et al., 2000 et LATTÉ, 2015. 
au cours des jours précédents ${ }^{17}$, soin aux animaux ${ }^{18}$, etc. Par ailleurs, la frontière entre espace et activités domestiques d'un côté, et espace et activités professionnelles de l'autre, est souvent poreuse sur les exploitations agricoles (MundLER, RÉmY, 2012). Les possibilités de contacts entre les résidents des exploitations et les pesticides qui y sont utilisés en sont d'autant multipliées : il n'est ainsi pas impossible que les produits épandus « dérivent » vers l'intérieur des habitations. De même, la manipulation de vêtements de travail souillés par les traitements lors des lessives reste, comme d'autres tâches domestiques, largement à la charge des épouses d'agriculteurs, même lorsque celles-ci sont salariées hors de l'exploitation (BESSIÈRE, 2004 ; RIEU, 2004).

Lors de l'assemblée générale de 2015 dédiée aux femmes qui soutiennent Phyto-victimes, outre des épouses et des filles d'agriculteurs malades ou décédés évoquant leur combat médico-administratif et politique, deux femmes scientifiques sont également invitées à venir présenter leurs travaux de recherche ${ }^{19}$. La première, Isabelle Baldi, épidémiologiste spécialiste des liens entre exposition aux pesticides et santé des travailleurs agricoles, souligne les limites des connaissances disponibles quant aux effets des pesticides sur la santé des femmes. Elle évoque certains biais des enquêtes épidémiologiques et déplore le peu d'attention qu'elles accordent aux expositions des agricultrices dans ou hors des champs agricoles ${ }^{20}$. La seconde, Nathalie Jas, qui a travaillé sur l'histoire des réglementations relatives à l'usage des pesticides, soulève la difficulté qu'il y a à penser certaines situations de travail plutôt féminisées comme risquées pour la santé : le lavage des combinaisons de protection utilisées lors des épandages ou encore la manipulation récurrente de végétaux sur lesquels des résidus de produits peuvent subsister. Au-delà des figures de l'épouse aidante ou de la femme endeuillée, largement mobilisées par les Phyto-victimes, les interventions de ces chercheuses mettent en avant d'autres figures de femmes, mères au foyer ou travailleuses agricoles, exposées aux produits. Ces interventions ne suscitent aucune question du public réuni ce soir-là, ni de véritable discussion parmi les membres de l'association le lendemain. De manière générale, l'absence de femmes atteintes de pathologies imputables aux pesticides dans le conseil d'administration de Phytovictimes ne semble pas soulever d'interrogation collective.

17. Une publication récente suggère que les niveaux d'exposition en ré-entrée dans les parcelles de vignes sont au moins aussi importants que ceux qui résultent de la manipulation des produits sur ces mêmes parcelles (BALDI et al., 2014).

18. Les activités d'élevage entraînent en effet une exposition aux pesticides utilisés comme médicaments antiparasitaires sur les animaux et comme biocides dans les locaux. À ce sujet, voir le rapport publié par l'Anses en 2016 intitulé Expositions professionnelles aux pesticides en agriculture, Maisons-Alfort, Anses, pp. 21-22 ; en ligne : https :// www.anses.fr/fr/system/files/AIR2011SA0192Ra.pdf, consulté le 9 mars 2017.

19. Nous entretenons des relations de travail avec ces deux chercheuses : la recherche présentée dans cet article s'inscrit dans un projet animé par Nathalie Jas ; l'un d'entre nous a participé trois ans durant à un groupe d'experts de l'Anses portant sur les expositions des travailleurs agricoles aux pesticides, auquel ces deux chercheuses ont également pris part. 20. L'un de ces biais est notamment la focalisation sur les activités de préparation et d'épandage des produits, généralement effectuées par des hommes. En revanche, les expositions occasionnées par le travail qu'une population généralement plus féminine accomplit sur les parcelles tout juste traitées, sont beaucoup moins étudiées (JACOBS, DiNHAM, 2003 ; HABIB et al., 2014). 
Ce silence souligne la domination au sein de Phyto-victimes d'une conception genrée de l'exposition aux pesticides, qui fait des maladies imputables à ces produits une affaire typiquement masculine. Il renvoie, plus largement, à une difficulté des exploitants membres de Phyto-victimes à verbaliser le problème de l'exposition aux pesticides des membres de leur foyer. Ils n'évoquent pas davantage les situations lors desquelles, par le passé, les membres de leur famille, ou eux-mêmes quand ils étaient enfants, ont pu être exposés. L'absence de ces thèmes ne provient en aucun cas d'un déni général quant au fait que leurs activités sont susceptibles d'endommager non seulement leur propre santé, mais également celles de tiers. Ces exploitants soulignent en effet volontiers leurs responsabilités vis-à-vis de la santé des riverains ou de celles des générations futures d'agriculteurs ${ }^{21}$. Il semble cependant qu'il leur soit plus difficile de parler des effets de l'activité agricole sur la santé des tiers les plus proches. Une telle difficulté peut être interprétée d'au moins deux manières complémentaires. D'une part, on peut faire l'hypothèse que l'exposition des proches occupe une place secondaire dans les représentations du risque qu'ont les agriculteurs rencontrés. Ils construisent en effet ces dernières à partir des informations les plus saillantes dont ils disposent. Ils ont ainsi une idée plus précise de leurs propres activités de travail que de celles de leurs proches dans la sphère domestique. De plus, les messages de prévention qu'ils reçoivent les concernent au premier chef puisqu'il y est question de préparation de bouillie et d'épandage, ou de figures non agricoles comme les riverains. Il n'est enfin pas non plus impossible qu'inconsciemment, les agriculteurs construisent leurs représentations des risques de manière à diminuer l'éventuel inconfort psychologique que susciterait la prise en compte des dangers liés à l'exposition des proches. D'autre part, on peut faire l'hypothèse que cette invisibilité procède moins d'une difficulté à se représenter l'exposition de la conjointe ou des enfants que d'une difficulté à énoncer cet enjeu, à en parler à un tiers, qu'il soit proche parent, collègue... ou sociologue. C'est vers une telle interprétation que l'extrait d'entretien suivant, réalisé avec Magalie, veuve d'agriculteur décédé des suites d'un cancer du sang, et sa fille, nous entraîne :

«Magalie : - Quand on en parlait entre nous, on disait : "On est sûrs que c'est ça [sous-entendu, on est sûrs que la maladie est liée à l'exposition à des pesticides]." [...] Entre nous, voilà, entre nous on le disait. Après, on ne le disait pas ailleurs. Entre nous, ce que je veux dire, devant nos copains, mais même [à] nos familles, on n'en parlait pas.

Fille : - Les parents, c'était compliqué parce qu'ils étaient agriculteurs, donc c'était aussi leur balancer leur responsabilité en travers de la tronche. Les vignes, ce sont eux qui les ont plantées. Il avait perdu sa sœur avant, d'un cancer, et il y a de fortes chances que justement ce soit dû... [...]

21. Depuis sa création, une grande part des actions de l'association Phyto-victimes consiste ainsi à organiser des conférences au sein d'établissements d'enseignement agricole pour informer les futures générations d'agriculteurs des dangers des pesticides pour leur santé. 
- Et puis Papi, on n'avait pas envie... il a 90 ans, on n'a pas envie de l'emmerder avec ça. »

(Entretien avec Magalie, membre du conseil d'administration de Phyto-victimes, veuve d'un exploitant décédé, en présence de sa fille, juillet 2012)

Cet entretien est le seul de notre enquête au cours duquel la personne interrogée évoque spontanément les effets passés des pesticides utilisés par les parents d'un agriculteur sur la santé de ce dernier. Il soulève la question de la responsabilité des générations passées ${ }^{22}$ et illustre, plus généralement, la difficulté qu'il peut y avoir à évoquer explicitement la question de l'exposition des proches aux pesticides, non seulement avec des tiers externes, mais aussi au sein même des familles concernées ${ }^{23}$.

\section{Deuil et engagement radical}

Les femmes ne sont cependant pas cantonnées au rôle d'épouses accompagnantes d'hommes souffrants au sein de Phyto-victimes. Plusieurs des femmes présentes au sein du conseil d'administration ont connu l'expérience du deuil. Deux épouses endeuillées, Magalie et Yvette, intègrent le conseil d'administration dès la création de l'association. Elles y sont rejointes, respectivement en 2014 et en 2015, par deux autres femmes endeuillées, l'une à la suite de la mort de son père viticulteur atteint d'un cancer du poumon, l'autre après le décès de son frère, salarié viticole souffrant d'un cancer des voies hépatiques. La place au sein de l'association de ces femmes n'ayant plus d'agriculteur malade à accompagner dans un parcours de reconnaissance apparaît particulièrement problématique. D'un côté, elle est centrale dans la mesure où elles font partie des membres qui s'expriment le plus au cours des réunions et participent régulièrement aux actions de l'association. Elles comptent aussi parmi ceux qui prennent le plus volontiers la parole en public. Les membres du conseil d'administration considèrent en effet que cette parole féminine et endeuillée est susceptible de convaincre des agriculteurs malades ou leur famille de franchir le pas et de se lancer ou bien dans le mouvement ou bien dans des démarches de reconnaissance en maladie professionnelle. Elles incarnent enfin de manière particulièrement tragique les conséquences des pesticides sur la santé humaine auprès de l'opinion et des pouvoirs publics. D'un autre côté, la participation des femmes endeuillées aux activités de

22. L'une des limites de notre enquête est de ne pas avoir interrogé les pères ou mères des agriculteurs malades. Si les entretiens ont eu le plus souvent lieu en présence du conjoint et/ou des enfants, un seul a été réalisé en présence des parents.

23. Il est probable que la conception genrée de l'exposition aux pesticides exposée ici évolue au cours du temps, par exemple à mesure de la répétition des interactions entre certains membres de Phyto-victimes et de chercheurs porteurs d'une conception élargie. À ce titre, l'engagement très récent de l'association, en tant que partenaire, dans un projet de recherche régional avec des chercheurs du laboratoire d'Isabelle Baldi pourrait avoir quelques effets intéressants à suivre. Intitulé « Former des collectifs de femmes d'agriculteurs à la détection des situations d'exposition aux pesticides de leur conjoint et de leur famille », ce projet part non seulement de l'hypothèse que les conjointes des agriculteurs jouent un rôle de " "force de rappel” sur les questions de préservation de la santé des agriculteurs », mais a également pour objectif de constituer un espace de réflexion sur le lien entre expositions professionnelles et expositions domestiques aux pesticides. 
l'association ne va pas sans heurt à terme. Ainsi, quand on observe la composition du conseil d'administration, on remarque que l'implication des agriculteurs malades reste très stable dans la durée alors que celle des femmes ayant perdu un être cher est caractérisée par un fort turnover. Parmi les quatre femmes endeuillées qui ont participé au conseil d'administration de Phyto-victimes depuis sa création, trois l'ont aujourd'hui quitté en raison de mésententes, notamment avec certains exploitants encore en activité.

Au-delà d'éventuelles inimitiés personnelles, ce turnover reflète des prises de position politiques contrastées et potentiellement conflictuelles. Les femmes endeuillées sont porteuses d'un discours nettement plus radical que celui des autres membres de l'association sur les évolutions techniques et économiques de l'agriculture. En particulier, le développement rapide de l'agriculture biologique serait selon elles la réponse principale aux risques liés à l'utilisation de pesticides de synthèse (encadré 4). Les trois d'entre elles qui ont quitté Phyto-victimes ont d'ailleurs adhéré à des associations qui, comme Générations futures, militent en ce sens. À l'inverse, les exploitants malades qui siègent au conseil d'administration défendent une ligne plus réformiste, considérant que le déploiement de l'agriculture biologique est une option envisageable, mais insistant aussi sur la complexité de sa mise en œuvre. Si tous ont entamé une réflexion sur la possibilité de leur propre conversion à l'agriculture biologique, ils continuent généralement d'utiliser des pesticides de synthèse et soulignent l'importance d'envisager d'autres solutions (amélioration des équipements servant à l'épandage et à la protection individuelle, diminution progressive et partielle de la consommation d'intrants chimiques, choix des produits les moins dangereux, etc.). Dans les débats qui traversent l'association, les femmes endeuillées sont également les plus favorables à une dénonciation frontale de l'insuffisance des mesures prises par les pouvoirs publics pour limiter les risques dus à l'utilisation des pesticides. L'une d'entre elles, éducatrice et fille d'un viticulteur bordelais, a par exemple récemment déposé une plainte au pénal, avec pour objectif de mettre en cause non seulement les stratégies des entreprises qui fabriquent ces produits, mais aussi l'inconséquence avec laquelle les services de l'État ont encadré leur mise en marché.

Les exploitants qui dirigent le conseil d'administration de Phyto-victimes mettent volontiers ces difficultés sur le compte des émotions et de la colère associées au deuil, qui expliquent selon eux l'adoption par ces femmes de points de vue radicaux compliquant à terme leur engagement dans l'association. Plutôt que cette interprétation, dont une version savante est proposée par la sociologie des « nouveaux mouvements émotionnels » (WALGRAVE, VERHULST, 2006), nos données de terrain nous conduisent à privilégier une analyse du deuil comme expérience de transformation des contextes familiaux et professionnels. Dans la lignée de recherches s'intéressant au lien entre l'expression des émotions dans les mouvements sociaux et les positions sociales de leurs membres (WEED, 1990 ; LATTÉ, 2015), nous remarquons en effet que, parmi les femmes endeuillées qui siègent ou ont siégé au conseil d'administration de Phytovictimes, seulement une est exploitante et qu'aucun de ses enfants ne projette de 


\section{ENCADRÉ 4}

\section{Du deuil à l'engagement pour l'agriculture biologique}

Fille de fonctionnaire, ce dont, selon elle, il ne faut « pas se vanter dans le milieu agricole », Magalie suit des études de comptabilité et occupe des emplois de secrétariat dans un lycée professionnel (non agricole) pendant plusieurs années. À la fin des années 1980, elle se met en couple avec Richard, agriculteur et éleveur charentais, qui a repris une petite exploitation familiale qu'il a progressivement agrandie et modernisée (troupeau de 130 vaches à viande, développement de la céréaliculture sur 60 hectares, et culture de parcelles de vigne). En 2002, les médecins lui diagnostiquent une leucémie. Pendant près de dix ans, Richard se bat contre la maladie qu'il fait reconnaître comme affection professionnelle en 2006. En 2011, à la mort de Richard, Magalie se retrouve à la tête de l'exploitation et gérante de la société agricole. Elle s'investit pour maintenir son bon fonctionnement et l'inscrire dans un processus de conversion à l'agriculture biologique. Elle s'appuie pour cela à la fois sur la bonne santé financière de l'exploitation et sur les encouragements de ses deux filles qui, bien que poursuivant des études de droit, l'aident parfois dans les travaux agricoles. Magalie s'implique également assidûment dans Phytovictimes à la création de l'association. Peu avant sa mort, son mari avait en effet rencontré son actuel président et s'était enthousiasmé à l'idée de constituer un collectif d'agriculteurs victimes des pesticides. Membre du conseil d'administration, présente à toutes les actions, Magalie démissionne cependant au bout de quelques années, reprochant à l'association de n'être pas assez clairement engagée dans la promotion de l'agriculture biologique.

poursuivre une activité agricole. Les femmes qui ont perdu leur époux agriculteur ne projettent pas leur avenir ou celui de leur famille dans le milieu agricole, ce qui autorise l'adoption de positions dénonciatrices vis-à-vis des acteurs qui ont promu l'intensification des pratiques agricoles. À l'inverse, pour les agriculteurs malades membres de Phyto-victimes, le maintien à flot de l'exploitation, voire l'éventuelle question de sa transmission aux générations suivantes, imposent des contraintes difficilement compatibles avec une dénonciation radicale du productivisme en général, et des pesticides en particulier. Expérience intime douloureuse, le deuil est aussi un déplacement social qui, en éloignant ceux qui le traversent du milieu agricole, compromet leur capacité à s'arranger durablement des compromis qui structurent l'action de Phyto-victimes ${ }^{24}$.

\footnotetext{
24. Pour illustrer que c'est sans doute moins l'appartenance au genre féminin que l'expérience du deuil qui apparaît déterminante dans cette difficulté à rester dans le giron de l'association, on rappellera que parmi les premiers membres du conseil d'administration se trouvait également un homme endeuillé, François, père d'un viticulteur décédé peu de temps après la création de l'association, et qui choisit lui aussi de quitter cette dernière au cours des années 2014-2015, en reprochant aux exploitants qui la dirigent leur manque de radicalité dans la dénonciation des pesticides.
} 
À travers le cas de l'association Phyto-victimes, notre article montre que certaines mutations contemporaines des familles d'agriculteurs, bien étudiées par la sociologie rurale, peuvent non seulement contribuer à fragiliser le mythe de l'unité paysanne mais également à mettre en débat les effets des choix technologiques productivistes historiquement promus par les politiques agricoles. Dotées de capitaux scolaires et sociaux de plus en plus variés, les conjointes d'agriculteurs mettent souvent tout en œuvre pour que leurs maris soient officiellement reconnus victimes des pesticides. Elles les aident à prendre conscience du lien entre la dégradation de leur état de santé et les produits qu'ils ont manipulés au cours de leur carrière, à entamer des démarches médico-administratives de reconnaissance de maladies professionnelles, à poursuivre parfois sur le terrain judiciaire cette quête de compensation, et à militer pour un contrôle plus strict des produits de l'industrie phytopharmaceutique. Ce constat mériterait toutefois d'être mis à l'épreuve d'une enquête qui prendrait davantage en compte les parcours victimaires interrompus ou jamais commencés, à partir de monographies familiales complètes, au-delà du recueil souvent incident dans notre propre travail empirique d'éléments relatifs aux proches parents des victimes rencontrées. Dans cette perspective, il serait notamment intéressant d'observer si certaines configurations familiales et techniques éloignées de celles observées dans cette étude - par exemple les petites exploitations de célibataires isolés, celles où tous les membres de la famille ont pour activité professionnelle principale un métier agricole, ou encore celles où des femmes dirigent une exploitation - constituent des contextes moins favorables à l'engagement dans de tels parcours.

Nous avons également montré que les conjointes occupent une place ambiguë dans le mouvement des victimes des pesticides, à la fois centrale et périphérique. Mises en avant comme d'indispensables relais d'une prise de conscience collective des dangers que font courir les pesticides aux travailleurs agricoles, elles sont moins reconnues - et ne se considèrent pas - comme des victimes potentielles alors qu'elles aussi sont parfois exposées aux produits de traitement utilisés. Celles qui ont fait l'expérience du deuil d'un conjoint ou d'un père, jouent un rôle prépondérant dans la cause des victimes des pesticides dans la mesure où, par leur histoire et la manifestation de leur douleur, elles incarnent de manière particulièrement forte les dangers de ces substances. En même temps, du fait de leur relatif éloignement vis-à-vis des enjeux productifs des exploitations, elles sont souvent porteuses d'une dénonciation radicale des dangers de l'agriculture intensive en général, et des pesticides en particulier, qui, à terme, peut les marginaliser dans ce mouvement. Plutôt qu'une invisibilisation du travail militant des femmes, le cas des Phyto-victimes rend observable une mise en visibilité partielle, centrée sur certains rôles sociaux (épouse accompagnatrice et/ou endeuillée) auxquels sont attachées certaines qualités (ténacité, sensibilité). Un tel processus gagnerait à être étudié au moyen d'une méthode d'enquête plus directement centrée sur l'expérience des femmes au sein de ce type d'action collective, notre propre travail reposant principalement sur des données qui, pour être nombreuses, n'en sont pas moins incidentes. 
La dynamique politique de l'association Phyto-victimes et les tensions qui la traversent, donnent à voir le couple comme « un espace où peuvent se négocier les points d'articulation entre agriculture et autres mondes sociaux » (DUBUISSON, GIRAUD, 2010, p. 111). Notre travail incite néanmoins à ne pas réduire la focale au seul couple pour comprendre le lien entre attachements familiaux et engagement politique. Il suggère notamment des pistes pour l'élargir à la question des relations intergénérationnelles, en particulier celles qui ont trait au destin social des enfants d'exploitants, et à celle de l'expérience du deuil et de la perte d'un proche qui peut être non seulement l'époux - ou l'épouse -, mais aussi le père ou la mère, le frère ou la sœur, ou encore le fils ou la fille. D'une manière générale, c'est bien l'ensemble des évolutions auxquelles le monde agricole est confronté qui constitue la toile de fond d'une mobilisation inédite portée par des agriculteurs se revendiquant d'une agriculture « conventionnelle » tout en construisant un discours critique à l'égard de cette dernière. De telles perspectives de recherche gagneraient à être explorées à travers d'autres figures de travailleurs indépendants pour lesquelles la contiguïté entre l'espace professionnel et l'espace domestique, tout comme l'enjeu de la transmission du métier entre générations, structurent fortement la vie familiale.

\section{BibliogRAPHIE}

ALARCON L. (2008), «"Maintenant, faut presque être ingénieur pour être agriculteur." Choix et usages des formations professionnelles agricoles dans deux familles d'agriculteurs », Revue d'études en agriculture et environnement, vol. 88, $\mathrm{n}^{\circ} 3$, pp. 95-118.

Baldi I., Lebailly P., Bouvier G., Rondeau V., Kientz-Bouchart V., Canal-Raffin M., GARRIGOU A. (2014), "Levels and Determinants of Pesticide Exposure in Re-Entry Workers in Vineyards: Results of the PESTEXPO Study", Environmental Research, vol. 132, pp. 360-369.

BARBot J., Fillion E. (2007), « La dynamique des victimes. Les formes d'engagement associatif face aux contaminations iatrogènes (VIH et prion) », Sociologie et sociétés, vol. 39, $\mathrm{n}^{\circ} 1$, pp. 217-247.

BARTHEZ A. (1982), Famille, travail et agriculture, Paris, Economica.

BESSIÈRE C. (2004), « "Vaut mieux qu'elle travaille à l'extérieur !” Enjeux du travail salarié des femmes d'agriculteurs dans les exploitations familiales », Cahiers du genre, ${ }^{\circ}$ 37, pp. 93-114.

BESSIÈrE C. (2010), De Génération en génération. Arrangements de famille dans les entreprises viticoles de Cognac, Paris, Raisons d'agir.

BESSIÈRE C. (2011), «Les séparations conjugales dans les familles agricoles », Informations sociales, $\mathrm{n}^{\circ} 164$, pp. 64-71.

Bessière C., GiRAud C., Renahy N. (2008), «Introduction - Famille, travail, école et agriculture », Revue d'études en agriculture et environnement, vol. 88, nº 1, pp. 5-19.

Brown P., Ferguson F. I. T. (1995), "Making a Big Stink: Women's Work, Women's Relationships, and Toxic Waste Activism", Gender and Society, vol. 9, n 2 2, pp. 145-172. 
BRunEAu I. (2007), « La Confédération paysanne : s’engager à “juste” distance », Ruralia, $\mathrm{n}^{\circ} 20$, pp. 219-230.

Bruneau I. (2013), « L'érosion d'un pouvoir de représentation. L'espace des expressions agricoles en France depuis les années $1960 »$, Politix, n 103, pp. 9-29.

ChAMPAGNe P. (2002), L'Héritage refusé. La crise de la reproduction sociale de la paysannerie française 1950-2000, [recueil de 6 articles publiés entre 1975 et 1987], Paris, Seuil.

Comer C. (2015), «Agricultrices ou médiatrices ? Prise en charge "féminine" de l'image de la profession et conservatisme agricole », Mouvements; en ligne : http ://mouvements. info/agricultrices-ou-mediatrices-prise-en-charge-feminine-de-limage-de-la-profession-etconservatisme-agricole/, consulté le 10 mars 2017.

Contamin J.-G. (2007), « Genre et modes d'entrée dans l'action collective. L'exemple du mouvement pétitionnaire contre le projet de loi Debré », Politix, n 78, pp. 13-37.

DÉCosse F. (2013), « Entre "usage contrôlé”, invisibilisation et externalisation. Le précariat étranger face au risque chimique en agriculture intensive », Sociologie du travail, vol. $55, \mathrm{n}^{\circ} 3$, pp. 322-340.

DESRIERS M. (2007), « L'agriculture française depuis cinquante ans : des petites exploitations familiales aux droits à paiement unique », in Insee, L'Agriculture, nouveaux défis, coll. «Insee Références », Paris, Insee, pp. 17-30.

Dubuisson S., GiRaud C. (2010), « Les agriculteurs entre clôtures et passerelles », in Hervieu B., Mayer N., Muller P., Purseigle F., Rémy J. (dir.), Les Mondes agricoles en politique : de la fin des paysans au retour de la question agricole, Paris, Presses de Sciences Po, pp. 111-129.

Dufour A., GiRAud C. (2012), « Le travail dans les exploitations d'élevage bovin laitier est-il toujours conjugal ?», Inra productions animales, vol. 25, n 2, pp. 169-180.

DunEZAT X. (2006), « Le traitement du genre dans l'analyse des mouvements sociaux : France/ États-Unis », Cahiers du genre, $\mathrm{n}^{\circ} 3$ (hors-série n ${ }^{\circ}$ 1), pp. 117-141.

Einwohner R. L., Hollander J. A., Olson T. (2000), "Engendering Social Movements: Cultural Images and Movement Dynamics”, Gender and Society, vol. 14, n ${ }^{\circ}$ 5, pp. 679-699.

Felstiner W. L. F., Abel R. L., Sarat A. (1980-1981), “The Emergence and Transformation of Disputes: Naming, Blaming, Claiming”, Law \& Society Review, vol. 15, n 3-4, pp. 631-654.

FiLlieule O. (2009), « Travail militant, action collective et rapports de genre », in Fillieule O., Roux P. (dir.), Le Sexe du militantisme, Paris, Presses de Sciences Po, pp. 23-72.

Garrigou A., BAldi I., Dubuc P. (2008), « Apports de l'ergotoxicologie à l'évaluation de l'efficacité réelle des EPI devant protéger du risque phytosanitaire : de l'analyse de la contamination au processus collectif d'alerte », Perspectives interdisciplinaires sur le travail et la santé, vol. 10, $\mathrm{n}^{\mathrm{o}} 1$; en ligne : https ://pistes.revues.org/2137, consulté le 10 mars 2017.

GiRAud C., RÉmy J. (2008), «Les choix des conjoints en agriculture », Revue d'études en agriculture et environnement, vol. 88, $\mathrm{n}^{\circ} 1$, pp. 21-46. 
GiRAud C., RÉmy J. (2013), «Division conjugale du travail et légitimité professionnelle. Le cas des activités de diversification agricole en France », Travail, genre et sociétés, $\mathrm{n}^{\circ} 30$, pp. 155-172.

GiRAud C., RÉmy J. (2015), « Être ou ne pas être agriculteur. Capital scolaire et devenir socioprofessionnel des fils d'agriculteurs », in Gasselin P., Choisis J.-P., Petit S., Purseigle F., Zasser S. (dir.), L'Agriculture en famille : travailler, réinventer, transmettre, Les Ulis, EDP Sciences, pp. 305-326.

Habib R. R., Hojeis S., Elzein K. (2014), “Gender in Occupational Health Research of Farmworkers: A Systematic Review”, American Journal of Industrial Medicine, vol. 57, n 12, pp. 1344-1367.

Hervieu B., Purseigle F. (2013), Sociologie des mondes agricoles, Paris, Armand Colin.

Jacobs M., Dinham M. (eds) (2003), The Silent Invaders: Pesticides, Livelihoods, and Women's Health, New York, Zed Books.

Jouzel J.-N., Dedieu F. (2013), « Rendre visible et laisser dans l'ombre. Savoir et ignorance dans les politiques de santé au travail », Revue française de science politique, vol. $63, \mathrm{n}^{\circ} 1$, pp. 29-49.

Jouzel J.-N., Prete G. (2013), « De l'intoxication à l'indignation. Le long parcours d'une victime des pesticides », Terrains et travaux, $\mathrm{n}^{\circ} 22$, pp. 59-76.

Jouzel J.-N., Prete G. (2014a), « Devenir victimes des pesticides. Le recours au droit et ses effets sur la mobilisation des agriculteurs Phyto-victimes », Sociologie du travail, vol. 56, $\mathrm{n}^{\mathrm{o}} 4$, pp. 435-453.

Jouzel J.-N., Prete G. (2014b), « Des patrons victimes de leurs conditions de travail ? Les exploitants agricoles face aux risques liés à l'utilisation des pesticides », in Célérier S. (dir.), Le Travail indépendant : statut, activités et santé, Rueil-Malmaison, Éditions Liaisons, pp. 225-238.

Jouzel J.-N., Prete G. (2015), « Mettre en mouvement les agriculteurs victimes des pesticides. Émergence et évolution d'une coalition improbable », Politix, n 111, pp. 175-196.

Krauss C. (1993), "Women and Toxic Waste Protests: Race, Class and Gender as Resources of Resistance", Qualitative Sociology, vol. 16, n 3, pp. 247-262.

LAGRAVE R.-M. (1983), « Bilan critique des recherches sur les agricultrices en France », Études rurales, $\mathrm{n}^{\circ} 92$, pp. 9-40.

LATTÉ S. (2015), « Des “mouvements émotionnels” à la mobilisation des émotions. Les associations de victimes comme objet électif de la sociologie des émotions protestataires », Terrains/ Théories, $\mathrm{n}^{\circ} 2$; en ligne : http://teth.revues.org/244, consulté le 10 mars 2017.

LORVELLEC L. (1998), « L'agriculteur “sous contrat” », in Supiot A. (dir.), Le Travail en perspectives, Paris, LGDJ, pp. 179-197.

Maresca S. (1983), Les Dirigeants paysans, Paris, Minuit.

Muller P. (1984), Le Technocrate et le paysan. Essai sur la politique française de modernisation de l'agriculture de 1945 à nos jours, Paris, Éditions de l'Atelier. 
Mundler P., RÉmy J. (2012), «L'exploitation familiale à la française : une institution dépassée ?», L'Homme et la société, n 183-184, pp. 161-179.

Perkins T. E. (2012), “Women's Pathways Into Activism. Rethinking the Women's Environmental Justice Narrative in California's San Joaquin Valley", Organization \& Environment, vol. 25, n ${ }^{\mathrm{o}}$ 1, pp. 76-94.

Purseigle F. (2004), Les Sillons de l'engagement. Jeunes agriculteurs et action collective, Paris, L'Harmattan.

RatTin S. (2002), « Deux jeunes ménages d'agriculteurs sur cinq ont des ressources non agricoles », in Insee, Données sociales, Paris, Insee, pp. 439-445.

RÉMY J. (1987), « La crise de professionnalisation en agriculture : les enjeux de la lutte pour le contrôle du titre d'agriculteur », Sociologie du travail, vol. 29, n 4, pp. 415-441.

RiEu A. (2004), «Agriculture et rapports sociaux de sexe. La "révolution silencieuse" des femmes en agriculture », Cahiers du genre, $\mathrm{n}^{\circ} 37$, pp. 115-130.

RIEU A., FrIC G. (2008), «Agricultrices et syndicalisme, quelles avancées ? », Pour, n 196197, pp. 230-237.

SAMAK M. (2013), «Quand la "bio" rebat les cartes de la représentation des agriculteurs. L'institutionnalisation de l'agriculture biologique dans les Alpes-Maritimes », Politix, $\mathrm{n}^{\circ}$ 103, pp. 125-148.

TAYLOR V. (1999), “Gender and Social Movements. Gender Processes in Women's Self-Help Movements", Gender and Society, vol. 13, n 1, pp. 8-33.

ThÉBAud-Mony A. (2006), « Histoires professionnelles et cancer », Actes de la recherche en sciences sociales, $\mathrm{n}^{\circ} 163$, pp. 18-31.

TraïNi C. (dir.) (2009), Émotions... mobilisation!, Paris, Presses de Sciences Po.

Walgrave S., Verhulst J. (2006), “Toward New Emotional Movements? A Comparative Exploration Into a Specific Movement Type”, Social Movement Studies, vol. 5, n 3, pp. 275-304.

Weed F. J. (1990), “The Victim-Activist Role in the Anti-Drunk Driving Movement", The Sociological Quarterly, vol. 31, n 3, pp. 459-473. 